Psychotherapeut 2021 $66: 132-139$ https://doi.org/10.1007/s00278-020-00470-6 Online publiziert: 5 . November 2020

(c) Der/die Autor(en) 2020

Carolin Fischer ${ }^{1,2} \cdot$ Annette Schröder ${ }^{1,2}$. Jens Heider ${ }^{2}$

'Arbeitseinheit für Klinische Psychologie und Psychotherapie des Erwachsenenalters, Universität KoblenzLandau, Landau, Deutschland

${ }_{2}^{2}$ Psychotherapeutische Universitätsambulanz, Universität Koblenz-Landau, Landau, Deutschland

\title{
Kognitive Verhaltenstherapie bei Autofahrangst
}

\section{Exposition im Straßenverkehr}

- Die Angst ist auf das Autofahren oder auf Gedanken daran beschränkt.

- Die Angst wird durch physiologische Symptome erlebt (vegetative Symptome, Symptome, die Thorax und Abdomen betreffen, psychische Symptome wie Schwindel oder Unsicherheit).

- Es bestehen eine deutliche emotionale Belastung und Einschränkung im Alltag durch die Symptome der Autofahrangst oder das Vermeidungsverhalten.

Allerdings lässt sich die Autofahrangst wegen der Komplexität der Symptome nicht immer eindeutig einer diagnostischen Kategorie zuordnen. Während oftmals eine spezifische Phobie des situativen Typus vorliegt, muss differenzialdiagnostisch geprüft werden, ob die Autofahrangst nicht besser durch eine andere (Angst-)Störung erklärt werden kann. Treten neben anderen für die Panikstörung relevanten Kriterien auch außerhalb der Fahrsituationen Panikattacken im Alltag auf, sollte das Vorliegen einer Panikstörung in Erwägung gezogen werden. Für den Fall einer Klassifikation der Autofahrangst im Rahmen einer Agoraphobie sollte entweder in weiteren, auch öffentlichen Verkehrsmitteln Angst auftreten, oder es sollten neben der Angst vor dem Autofahren weitere Situationen wie offene Plätze, geschlossene Räume (Geschäfte, Kinos) oder Menschenmengen angstbesetzt sein oder gemieden werden. Steht dagegen die Angst, sich im Straßenverkehr peinlich oder unbeholfen zu verhalten und somit durch andere Verkehrsteilnehmende kritisiert zu werden, im Vordergrund, ist das Vorliegen einer sozialen Phobie zu prüfen. Auch im Rahmen einer posttraumatischen Belastungsstörung können, typischerweise infolge von Verkehrsunfällen, Autofahrängste und phobische Vermeidung gezeigt werden. Zusätzlich kann die Diagnose einer Zwangsstörung erwogen werden, wenn die Betroffenen infolge von Zwangsgedanken, beispielsweise unbemerkt einen Unfall verursacht oder Passanten verletzt zu haben, das Autofahren phobisch vermeiden oder unter sich aufdrängenden Zwangsimpulsen, wie dem wiederkehrenden Prüfen der gefahrenen Strecke, leiden.

Festzuhalten ist, dass die Autofahrangst in allen Fällen eine die Freiheit und Unabhängigkeit einschränkende Angst ist, die die Verfolgung privater, sozialer und beruflicher Interessen erschweren oder gar unmöglich machen kann. Für Betroffene ist das Fahren nur unter großem Stress- und Angsterleben möglich, wobei das Ausmaß der Störung von der Vermeidung spezifischer Fahrsituationen (z.B. Autobahnfahrten, Fahren durch Tunnel oder bei Nacht) bis zur kompletten Aufgabe des Autofahrens variieren kann (Taylor et al. 2002).

\section{Ursachen}

Autounfälle mit Kontrollverlusterfahrungen werden weitläufig als Ursache von Autofahrangst angenommen. Tatsächlich zeigen Studien, dass rund $70-80 \%$ der befragten, von Autofahrangst Betroffenen ein kritisches Ereignis im Auto angeben können (z.B. spontane Panikattacke am Steuer, Beinahe-Unfall; 
Ehlers et al. 1994; Munjack 1984). Auch indirekt, durch die Beobachtung brenzliger Verkehrssituationen oder von Verkehrsunfällen, können Autofahrängste bedingt sein. Rund ein Viertel der Befragten gibt bei der Frage nach den Ursachen an, dass sie schon immer ängstliche Autofahrer/-innen gewesen seien, während rund $10 \%$ keine Erklärung für die Entwicklung der Autofahrängste haben (Taylor und Deane 1999; Taylor et al. 1999).

Mangelnde Fahrerfahrung oder reduziertes Selbstvertrauen in die eigene Fahrfähigkeit kann eine Rolle bei der Entstehung von Fahrangst spielen. Betroffene zeigen höhere Fahrvermeidung und dysfunktionale autofahrbezogene Kognitionen. Aus kognitiver Perspektive können bei Autofahrangst verschiedene Angstfoci unterschieden werden, die entweder die Angst vor Unfällen, Verletzung und Kontrollverlust betreffen („danger expectancy“) oder die Angst vor dem Aufkommen der die Fahrfähigkeit einschränkenden Angstsensationen auf körperlicher und emotionaler Ebene hervorheben („anxiety expectancy“; Taylor et al. 2000). Diesen spezifischen dysfunktionalen Kognitionsfaktoren wird im vorgeschlagenen Behandlungsvorgehen besondere Beachtung zuteil, um einen jeweils darauf zugeschnitten Behandlungsansatz vorzuschlagen.

\section{Psychometrische Erfassung}

International liegen einige Selbstberichtsfragebogen vor, die autofahrbezogene Ängste erfassen: So z.B. der Accident Fear Questionnaire (AFQ; Kuch et al. 1985), der Travel Phobia Beliefs Questionnaire (TPBQ; Ehring et al. 2006), und der Safety Behaviors Questionnaire (SBQ; Ehring et al. 2006), die Unfallangst, dysfunktionale Grundannahmen nach Autounfällen und daraus resultierendes Sicherheitsverhalten erheben. Der Driving Situations Questionnaire (DSQ; Ehlers et al. 1994) erfasst das Ausmaß der Angst und Vermeidung in über 40 verschiedenen Fahrsituationen. Als bisher einziges in die deutsche Sprache übersetztes und validiertes Messinstrument ist der Driving Cognitions Questionnaire (DCQ; Heider et al.
2018) zu nennen. Der DCQ ermittelt autofahrbezogene Angstkognitionen und setzt sich aus den 3 Faktoren der panikbezogenen Sorgen (z.B. „Ich werde Schwierigkeiten haben $\mathrm{zu}$ atmen“), der unfallbezogenen Ängste (z. B. „Ich werde verletzt werden“) und der Angst vor der Bewertung durch andere (z. B. „Ich könnte durch Mitmenschen Kritik erfahren") zusammen. Es liegen Befunde für eine gute Reliabilität und Validität vor. In der therapeutischen Praxis kann der DCQ zur individualisierten Therapieplanung, insbesondere zur Bestimmung des Fokus der Autofahrangst, eingesetzt werden. Damit gibt der DCQ im Einzelfall Hinweise zur kognitiven Umstrukturierung relevanter dysfunktionaler Autofahrangstkognitionen und kann für die Auswahl aufzusuchender Situationen im Rahmen der Expositionen mit dem Ziel der Generierung von Erwartungsverletzungen eine gute Grundlage darstellen.

Da bis dato kein Screeninginstrument zu Diagnostik und Erfassung der Intensität von Autofahrangst vorlag, wurde kürzlich durch die Autoren das Instrument zur Erfassung der Autofahrangst entwickelt (IAA; Fischer et al., under review). Das aus 5 Items bestehende Instrument misst die Autofahrangst in Anlehnung an die Kriterien der spezifischen Phobie des DSM-5 auf einer 4-stufigen Antwortskala von 0 („trifft überhaupt nicht $\mathrm{zu}^{\prime}$ ) bis 3 („trifft voll und ganz $\left.\mathrm{zu}^{\prime \prime}\right)$ :

- Item 1: „Ich habe Angst vor dem Autofahren“ (subjektiv erlebtes Ausmaß an Angst vor dem Autofahren).

- Item 2: „Bei dem Gedanken, Auto zu fahren, male ich mir aus, was alles passieren könnte" (kognitive Ebene der Angstreaktion und Erwartungsangst).

- Item 3: „Wenn ich Auto fahre, spüre ich die Angst körperlich (z. B. Herzrasen, Schwitzen, Zittern, Engegefühl in der Brust, muskuläre Anspannung o. Ä.)“ (physiologische Angstreaktion).

- Item 4: „Ich vermeide das Autofahren“ (Vermeidungsverhalten).
- Item 5: „Meine Angst vor dem Autofahren belastet mich“ (Leidensdruck durch die Autofahrangst).

Das eindimensionale IAA weist eine gute interne Konsistenz von $\alpha=0,88$ auf. $\mathrm{Ab}$ einem „Cut-off“-Wert von 4 kann von einer klinisch-relevanten Autofahrphobie ausgegangen werden (Fischer et al., under review).

\section{Interventionsstudien zur kognitiven Verhaltenstherapie der Autofahrangst}

Kognitive Verhaltenstherapie und expositionsbasierte Intervention haben sich bei spezifischen Phobien in zahlreichen randomisierten kontrollierten Studien als hochwirksam erwiesen (z. B. Ruhmland und Margraf 2001). Für die Behandlung spezifischer Ängste vor dem Autofahren liegen bis dato lediglich Einzelfallstudien und Untersuchungen mit nichtrandomisierten Designs und geringer Fallzahl vor.

Eine Übersicht über existierende Interventionsstudien geben Fischer et al. (2020), worin kognitiv-behaviorale Therapieansätze, einschließlich technikgestützter Vorgehensweisen (z. B. virtuelle Realität und Fahrsimulatoren), traumafokussierte und hypnosebasierte Behandlungsansätze dargestellt werden (Fischer et al. 2020). Erste Hinweise auf die Wirksamkeit der genannten Therapieansätze liegen vor. So wurde gezeigt, dass durch die verhaltenstherapeutische Konfrontation mit Autofahrsituationen Habituation eintreten kann und korrigierende Erfahrungen generiert werden können (Horn et al. 2015; Townend und Grant 2006).

Erwartungsverletzungen im Sinne der Widerlegung einer dysfunktionalen autofahrbezogenen Annahme (z. B. „Sobald ich auf die Autobahn auffahre, werde ich nicht mehr klar denken und handeln können“) können zur Abnahme der Angst führen. Technische Hilfsmittel (z. B. Mobiltelefon mit Freisprechanlage) scheinen hilfreich zur Förderung der Bereitschaft zur In-vivo-Exposition, und die Nutzung von Fahrsimulatoren führte zur deutlichen Reduktion der Fahrangst (Kaussner et al. 2020). Die Nutzung der virtuellen 
Realität wird bei der Behandlung von Autofahrangst zunehmend in den Fokus gerückt. So kann eine gefahrenfreie Konfrontation erfolgen, deren Wirkung in Einzelfallstudien und ersten Pilotstudien nachgewiesen werden konnte (da Costa et al. 2018; Kaussner et al. 2020). Die Verfügbarkeit der dafür benötigten Technik beschränkt sich bisher jedoch auf ausgewählte Therapiezentren, und die Übertragbarkeit der in dervirtuellen Realität gemachten Erfahrung auf den Alltag der Patienten/Patientinnen muss in größeren Studien weitergehend geprüft werden. Zudem stellt sich die Frage, ob eine ausschließlich im virtuellen Raum stattfindende Expositionsbehandlung eine In-vivo-Exposition vollständig ersetzen kann, oder ob zum Transfer des Erlernten auf die reale Fahrsituation zusätzliche In-vivo-Expositionen erforderlich sind. Zur Höhenphobie liegen erste Erkenntnisse vor, die auf eine vergleichbar hohe Wirksamkeit der virtuellen Konfrontationsbehandlung hindeuten wie der Goldstandard der In-vivoExposition (Emmelkamp et al. 2001).

Da bei Autofahrangst neben der emotionalen Komponente der Angst außerdem die Komponenten der Kompetenz und Performanz von Bedeutung sind, sollten zukünftige Studien die Effektivität der verschiedenen Expositionsansätze vergleichen.

\section{Versicherungsrechtliche Aspekte bei Expositionen im Straßenverkehr}

Kommen Therapeut/-in und Patient/in gemeinsam zu der Entscheidung, eine vorliegende Autofahrangst mithilfe der In-vivo-Exposition zu behandeln, gilt es, einige die Umsetzung betreffende Aspekte zu beachten. Unklarheiten über rechtliche und versicherungstechnische Aspekte bei Expositionsübungen außerhalb der Praxisräume und im Straßenverkehr stellen für viele Therapeuten/ Therapeutinnen häufig eine Barriere bei der Durchführung von Expositionen dar (Pittig und Hoyer 2017). Die folgende zusammenfassende Darstellung rechtlicher Hintergründe bei der Exposition im Straßenverkehr dient der allgemeinen Information und kann keine verbindli- che Rechtsauskunft darstellen. Sie soll Anregungen bei der Planung der autofahrbezogenen Expositionen geben und zur Reflexion des eigenen professionellen Handelns anregen. Dennoch können im individuellen Fall juristische Fachpublikationen oder neue Rechtsprechungen $\mathrm{zu}$ abweichenden Ergebnissen führen.

Aufgrund der nicht gänzlich auszuschließenden Gefahr, die von Expositionen im Straßenverkehr ausgeht, ist die Beachtung rechtlicher Aspekte und Haftungsfragen von großer Bedeutung. So sind Therapeuten/Therapeutinnen dazu verpflichtet, fachgerecht, nach anerkannten Regeln der psychotherapeutischen Wissenschaft und Praxis zu behandeln. Voraussetzungen für die Invivo-Exposition sind die sachgerechte Aufklärung des/der Patienten/Patientin über die Risiken im Straßenverkehr, seine Einwilligung sowie die deren Dokumentation (Halbe 2003; Tintner 2002). Expositionsübungen im Straßenverkehr dürfen die Fahrtüchtigkeit nicht einschränken. Dennoch kann diese, unter phobischen Angstreaktionen, herabgesetzt sein, wenn Betroffene impulsiv handeln, indem sie beispielsweise abrupt die Autobahn verlassen oder Fahrstreifen wechseln, um gefürchteten Situationen („flight“) zu entkommen. Auch kann unter Angst die Konzentration reduziert sein („freeze").

Expositionen im Straßenverkehr sollten somit zunächst im Fahrschulauto in Begleitung eines Fahrlehrers stattfinden, damit die Fahrfähigkeit unter Angst geprüft sowie durch ein Fremdund Expertenurteil des/der Fahrlehrers/ Fahrlehrerin bestätigt werden kann. Liegt diese vor, kann der/die Therapeut/-in bei therapeutisch begleiteten Expositionen als Beifahrer/-in im patienteneigenen Auto mitfahren. Es ist eine sorgfältige Absprache zwischen Fahrlehrer/-in und Therapeut/-in zu empfehlen, um den verschiedenen Anforderungen der Situation (Konfrontation, Fertigkeitstraining und Einschätzung der Fahrfähigkeit) gerecht werden zu können. Insbesondere sollte allein der/die Therapeut/-in die Exposition anleiten, während sich hingegen der/die Fahrlehrer/-in auf ggf. notwendige Instruktionen zu Autofahrfertigkeiten fokussiert. Beruhigende oder bagatelli-
Psychotherapeut 2021 $\cdot 66: 132-139$ https://doi.org/10.1007/s00278-020-00470-6 (c) Der/die Autor(en) 2020

\section{Fischer · A. Schröder · J. Heider}

Kognitive Verhaltenstherapie bei Autofahrangst. Exposition im Straßenverkehr

\section{Zusammenfassung}

Die Exposition im Straßenverkehr bei der kognitiv-verhaltenstherapeutischen Behandlung der Autofahrangst ist oftmals mit Fragen der praktischen Umsetzung sowie mit Haftungsfragen verbunden. Die folgende Handlungsempfehlung gibt einen Überblick über die Diagnostik, Ätiologie und psychometrische

Erfassung der Autofahrangst und stellt versicherungsrechtliche Aspekte, die bei der Exposition im Straßenverkehr zu beachten sind, dar. Es wird ein Therapiemodul zur Behandlung der Autofahrangst vorgestellt, das konkrete Hinweise zur praktischen Umsetzung der Behandlung von Autofahrangst gibt.

\section{Schlüsselwörter}

Motivation · Psychoedukation · Habituation . Psychometrie - Rechtliche Aspekte

\section{Cognitive behavioral therapy of driving fear. Exposure in road traffic}

\section{Abstract}

Exposure to road traffic, as a part of cognitive behavioral therapy of driving phobia, is often related to uncertainty and pending questions regarding practical realization and insurance. The following guidelines outline diagnostic and etiological features and psychometric measures of driving phobia. Insurance legal issues relevant to exposure to road traffic are presented. A treatment module for driving phobia is outlined that gives concrete indications for the practical implementation.

Keywords

Motivation - Psychoeducation - Habituation . Psychometrics $\cdot$ Legal aspects

sierende Äußerungen durch den/die Fahrlehrer/-in während der Exposition sollten, auch wenn gut gemeint, unterlassen werden. Eine expositionsbasierte Autofahrangstbehandlung ohne den Einbezug eines/einer Fahrlehrers/ Fahrlehrerin und dessen Expertenur- 
teil ist aus Gründen der Sicherheit und Versicherung nicht zu empfehlen.

Weiterhin sollten die Übungen graduiert stattfinden, sodass die Fahrtüchtigkeit stets erhalten bleibt (Halbe 2003; Tintner 2002). Besonderes Augenmerk ist auf die Unplanbarkeit von Verkehrssituationen zu richten, da sich das Angstniveau und die Schwierigkeit der Übung durch unerwartetes Eintreten von z.B. Verkehrshindernissen, Sperrungen oder Staus verändern können. Mit dem/der Patienten/Patientin ist vorab zu besprechen, dass im Fall klinisch bedeutsamer Panikattacken im Straßenverkehr das Fahrzeug kontrolliert anzuhalten ist, um eine Eigen- und Fremdgefährdung auszuräumen. Im Fahrschulauto ist im Sinne der Konfrontation mit intensiven Angstsymptomen ein längeres Verbleiben in der stark angstbesetzten Fahrsituation durch die Möglichkeit des Eingreifens des/der Fahrlehrers/Fahrlehrerin vertretbar. Im patienteneigenen Auto sollte einer starken, die Verkehrssicherheit einschränkenden Angst mit dem Anhalten des Autos und dem Abklingenlassen der Angstsymptomatik begegnet werden, um die sichere Weiterfahrt bei wiederhergestellter Fahrtüchtigkeit zu gewährleisten. Auch wenn diese Maßnahme zeitweise zur Verstärkung von Sicherheitsverhalten führen kann, ist sie bei der Exposition im patienteneigenen Auto unerlässlich.

Da private Fahrzeuge der Behandler zu Expositionszwecken bei nichtexpliziter Anmeldung zweckwidrig genutzt würden und dies zu einer Leistungsfreistellung des Versicherers führen kann, wird empfohlen, das patienteneigene Auto für die Expositionen zu nutzen. Wenngleich den Autoren bis dato keine Verkehrsunfälle im Rahmen von Expositionen im Straßenverkehr bekannt sind, können solche nie ausgeschlossen werden. Dabei kommt eine beitragsmäßig unbegrenzte Haftung des Behandlers infrage, sofern eine Verletzung der Sorgfaltspflicht bei Planung und Durchführung der Expositionsbehandlung vorliegt. Der Abschluss einer entsprechenden Berufshaftpflichtversicherung schafft Abhilfe. Jeder/jede ambulante Psychotherapeut/-in hat über eine Berufshaftpflichtversicherung $\mathrm{zu}$ verfügen, die Schäden bei Behandlungs- und Aufklärungsfehlern (z. B. versäumte Dokumentation der Aufklärung und Einwilligung) abdeckt. Besonderer Beachtung muss dabei jedoch der „Benzinklausel“ geschenkt werden; diese schließt Schäden, die durch die Nutzung eines Autos zur Expositionsbehandlung entstanden sind, aus. Hierzu wird die rechtzeitige Prüfung des Versicherungsvertrags empfohlen, der in einigen Fällen die Nutzung von motorisierten Fahrzeugen ausschließt (Deutsche Psychotherapeuten Vereinigung 2016; Halbe 2003; Tintner 2002).

Im Schadensfall sind Ansprüche des/der Patienten/Patientin gegenüber seinem/ihrem Behandler/-in zu beachten, da die gesetzliche Kraftfahrzeughaftpflichtversicherung bei einem Behandlungs- oder Aufklärungsfehler nicht zum Tragen kommt. Eine zusätzliche Insassenunfallversicherung kommt zumeist für entstandene Schäden des/der Patienten/Patientin als Fahrzeugführer/-in ebenfalls nicht auf, da gewöhnlich ein Ausschluss für Unfälle vorgesehen ist, die durch Geistes- und Bewusstseinsstörungen des/der Fahrers/Fahrerin verursacht wurden. (Regress-)Ansprüche der Versicherung gegen den Behandelnden können gestellt werden, wenn sie Ansprüche Geschädigter beglichen hat und sich anschließend die Frage nach therapeutischer (Mit-)Schuld stellt (Halbe 2003; Tintner 2002).

Ansprüche geschädigter Dritter gegen den Behandelnden werden zumeist über die Kraftfahrzeughaftpflichtversicherung durchgesetzt. Die Versicherung ist auch bei zweckwidriger Verwendung des Fahrzeugs oder einem Mitverschulden vollständig einstandspflichtig (Halbe 2003; Tintner 2002).

Abschließend kann festgehalten werden, dass nach erfolgter Aufklärung und sorgfältiger Dokumentation eine graduierte Exposition im Straßenverkehr erfolgen kann, wobei der Einbezug einer Fahrschule mit fachmännischer Fahrtauglichkeitseinschätzung ratsam ist, bevor das patienteneigene Auto genutzt wird. Die zu beachtenden versicherungsrechtlichen Aspekte sollten nicht zum Zurückschrecken vor Expositionen im Straßenverkehr führen. Das Ausbrechen aus dem gewohnten Therapiesetting und die beobachtbaren Erfolge sind für $\mathrm{Pa}$ tienten/Patientinnen und Therapeuten/ Therapeutinnen gleichermaßen zufriedenstellend und motivierend.

\section{Therapiemodul}

\section{Ziele und Struktur}

Im Folgenden wird ein Therapiemodul zur expositionsbasierten verhaltenstherapeutischen Behandlung von Autofahrangst vorgestellt, das parallel die Wirkmechanismen der Habituation durch Exposition, Umstrukturierung angstverstärkender Kognitionen und Generierung von Erwartungsverletzungen verfolgt. Ziele bei der Entwicklung des Moduls waren einerseits die Minimierung der Risiken einer Exposition im Straßenverkehr und andererseits die Berücksichtigung der Heterogenität in der Entstehung und Aufrechterhaltung der Autofahrängste. Zur differenziellen Indikation werden im Modul die bei Autofahrangst verschiedenen, im Vordergrund stehenden Angstfoci und zentralen Befürchtungen der panikbezogenen Sorgen, unfallbezogene Sorgen und Sorgen vor der Bewertung durch andere herangezogen. Das Modul findet damit unabhängig davon, in welcher Diagnose die Autofahrangst aufgeht, Anwendung. Das Therapiemodul umfasst 12 Doppelsitzungen (• Tab. 1) und wird aktuell im Rahmen einer Pilotstudie an der Universität Koblenz-Landau evaluiert (Fischer et al. in Vorbereitung).

\section{Diagnostikphase}

Nach sorgfältiger Anamneseerhebung, durchgeführter störungsspezifischer Testdiagnostik und Erarbeitung einer Mikroanalyse mit dem/der Patienten/ Patientin werden in der Diagnostikphase die gesammelten Ergebnisse der klinischen und statistischen Urteilsbildung rückgemeldet. Der Behandlungsplan, bestehend aus Psychoedukation, Vermittlung spezifischer kognitiv-behavioraler Kompetenzen zu Motivierung und Vorbereitung auf die Exposition sowie die graduierte Exposition werden erläutert. 
Tab. 1 Ablauf der kognitiv-verhaltenstherapeutischen Autofahrangstbehandlung

\begin{tabular}{|c|c|c|}
\hline Therapiephase & Sitzungen & Therapieziel und -methoden \\
\hline \multirow[t]{4}{*}{ Diagnostik } & \multirow[t]{4}{*}{$1-4$} & $\begin{array}{l}\text { Exploration und Anamnese der Autofahrängste, Herausarbeitung von Sicherheits- und Vermeidungsverhalten und } \\
\text { kognitiven Verzerrungen }\end{array}$ \\
\hline & & Durchführung von Verhaltensanalysen \\
\hline & & Durchführung der psychometrischen Diagnostik, z. B. DCQ, IAA \\
\hline & & Differenzialdiagnostische Einordnung der Autofahrangst \\
\hline \multirow[t]{4}{*}{ Psychoedukation } & \multirow[t]{4}{*}{$5-8$} & Psychoedukation zur Autofahrangst (Teufelskreis der Autofahrangst, Vermeidungsverhalten etc.) \\
\hline & & $\begin{array}{l}\text { Ableitung eines individuellen Störungsmodells der Autofahrangst mit Auswahl und Fokussierung eines besonders } \\
\text { relevanten Ätiologiefaktors } \\
\text { 1. Panikbezogene Ängste vor Körpersymptomen beim Fahren } \\
\text { 2. Unfallbezogene Sorgen und Ängste vor Kontrollverlust, Verletzung, Unfällen } \\
\text { 3. Ängste vor negativer Bewertung durch andere, Angst vor peinlichem, unbeholfenem Verhalten im Straßenverkehr }\end{array}$ \\
\hline & & Erläuterung der Rationale der Konfrontation im Straßenverkehr \\
\hline & & Erstellung einer Angsthierarchie von Autofahrängsten \\
\hline \multirow[t]{2}{*}{$\begin{array}{l}\text { Vermittlung } \\
\text { spezifischer } \\
\text { Kompetenzen zur } \\
\text { Vorbereitung auf } \\
\text { die Exposition }\end{array}$} & \multirow[t]{2}{*}{9 und 10} & $\begin{array}{l}\text { Bearbeitung des jeweils besonders relevanten Ätiologiefaktors und Erarbeitung spezifischer Kompetenzen zur } \\
\text { kognitiv-behavioralen Vorbereitung auf die Expositionsphase } \\
\text { 1. Panikbezogene Ängste: interozeptive Exposition mit Körpersymptomen, Korrektur der Fehlinterpretationen von } \\
\text { Körpersensationen und Auswirkungen auf Fahrfähigkeit } \\
\text { 2. Unfallbezogene Sorgen und Ängste: Korrektur von unfallbezogenen Sorgen, Wahrscheinlichkeitsüberschätzungen } \\
\text { von Unfällen und Diskriminationstraining zur Unterscheidung zwischen etwaiger vergangener Unfallsituation und } \\
\text { aktueller Fahrsituation im Hier und Jetzt } \\
\text { 3. Auf Bewertung durch andere bezogene Sorgen: Bearbeitung von dysfunktionaler Angst vor der Bewertung durch } \\
\text { andere, Verhaltensexperimente }\end{array}$ \\
\hline & & $\begin{array}{l}\text { Einführung in die Prinzipien der Achtsamkeit zur Lenkung des Aufmerksamkeitsscheinwerfers (Aufmerksamkeits- } \\
\text { lenkung nach innen vs. auf den Straßenverkehr) }\end{array}$ \\
\hline \multirow[t]{3}{*}{$\begin{array}{l}\text { Motivierung zur } \\
\text { Exposition }\end{array}$} & \multirow[t]{3}{*}{11 und 12} & $\begin{array}{l}\text { Gedankenexperiment: „Zu Ende denken“ einer phobischen Autofahrsituation zur Ableitung des Habituationsprin- } \\
\text { zips und der Konfrontationsrationale }\end{array}$ \\
\hline & & Einführung des Konzepts der Erwartungsverletzung und Erwartungsüberprüfungen \\
\hline & & Aufklärung und Einwilligung: In-vivo-Exposition, Risiken bei Teilnahme am Straßenverkehr \\
\hline \multirow[t]{3}{*}{ Exposition } & \multirow[t]{3}{*}{$13-20$} & Ggf. Exposition im Fahrsimulator \\
\hline & & $\begin{array}{l}\text { In-vivo-Exposition im Fahrschulauto mit Begleitung des Fahrlehrers sowie Fahrtauglichkeitseinschätzung durch } \\
\text { den Fahrlehrer }\end{array}$ \\
\hline & & In-vivo-Expositionen im patienteneigenen Auto \\
\hline \multirow{5}{*}{$\begin{array}{l}\text { Abschluss und } \\
\text { Booster-Sitzungen } \\
\text { in ein- bis } \\
\text { zweimonatlichen } \\
\text { Abständen }\end{array}$} & \multirow[t]{5}{*}{$21-24$} & Rückblick auf die Behandlung und Validierung der Fortschritte \\
\hline & & Umgang mit Rückschritten und Rückfallprophylaxe \\
\hline & & Planung weiterer unbegleiteter Expositionen anhand eines Übungsplans \\
\hline & & Reflexion der Lernerfahrungen aus selbstständig durchgeführten Expositionen \\
\hline & & Therapieevaluation \\
\hline
\end{tabular}

\section{Psychoedukation}

Im Rahmen der Psychoedukation schließt sich die Erarbeitung eines individuellen Erklärungsmodells an, wobei die Ergebnisse einer Mikroanalyse in Kombination mit einer Makroanalyse und aktuellen psychosozialen Belastungsfaktoren betrachtet werden. An dieser Stelle schlägt das Behandlungsmodul je nach im Vordergrund stehender Facette der Autofahrangst 3 Schwerpunktsetzungen bei der Entwicklung des Erklärungsmodells vor. Das Erklärungsmodell $\mathrm{zu}$ einer im Vordergrund stehenden panikbezogenen Angst fokussiert die Wahrnehmungs- und Bewertungsprozesse bei belastenden Körpersymptomen am Steuer. Im Fall der unfallbezogenen Ängste stehen die Herausarbeitung der Verarbeitungsprozesse nach etwaig erlebten Verkehrsunfällen bzw. die Erwartungsangst bei antizipierten Unfällen im Fokus. Die Angst vor Kontrollverlust, körperlicher Verletzung und möglichen Unfällen wird im Erklärungsmodell besonders berücksichtigt. Ist die Facette der Angst vor der Bewertung durch andere vordergründig, wird analog zu sozialphobischen Aufmerksamkeitsprozessen die aus der nach innen gerichteten Aufmerksamkeit resultierende Angst beleuchtet. Bewertungsprozesse, die zu Angst vor peinlichem und unbeholfenem Verhalten im Straßenverkehr führen und die Autofahrangst aufrechterhalten, werden betrachtet.

Im nächsten Schritt folgt Psychoedukation zu den Themen Angst im Allgemeinen und Autofahrangst im Speziellen, wobei körperliche Prozesse, erklärt durch Sympathikus und Parasympathikus, und 
die Funktion von Angst erläutert werden. Das Zusammenwirken von Kognition, Emotion, Verhalten und Physiologie wird anhand des Angstteufelskreises erarbeitet. Das Behandlungsmodul sieht vor, Sicherheits- und Vermeidungsverhaltensweisen des/der Betroffenen herauszuarbeiten (z. B. Vermeidung von Autobahnen, Fahren nur mit Beifahrern, Vermeidung von Linksabbiegen). Somit führt es in die Rationale der Konfrontation zur Generierung korrigierender Erfahrungen und Erwartungsverletzungen ein. Eine Angsthierarchie zur Vorbereitung der graduierten Exposition wird gemeinsam mit dem/der Betroffenen erarbeitet.

\section{Vermittlung von Kompetenzen zur Vorbereitung auf die Expositionsphase}

Anschließend werden individuell spezifische Kompetenzen zur kognitiv-behavioralen Vorbereitung auf die Expositionsphase vermittelt und trainiert. Hierbei besteht das Ziel jeweils in der Generierung von Erwartungsverletzungen (z. B. „Ich werde nicht ohnmächtig, wenn ich starke Körpersensationen erlebe.“ „Es passiert nicht immer ein Unfall, sobald ich über $100 \mathrm{~km} / \mathrm{h}$ schnell fahre.“ „Die Hinterherfahrenden hupen nicht, wenn ich nicht unmittelbar bei grün' an der Ampel losfahre.") und kognitiver Umstrukturierung.

Liegt bei der Person der Schwerpunkt auf den panikbezogenen Ängsten, werden Übungen zur interozeptiven Exposition vorgeschlagen, sodass die Auseinandersetzung mit der negativen Bewertung von Körpersensationen angeregt wird (Lang et al. 2018).

Wenn unfallbezogene Sorgen und Ängste im Vordergrund stehen, sollen die im Fall des Autofahrens nie gänzlich auszuschließende Möglichkeit einer Gefahrentstehung im Straßenverkehr erörtert sowie die Vor- und Nachteile des Inkaufnehmens dieses Risikos betrachtet werden. Eine realistische Gefahrenerwartung stellt das Ziel dar. Auch wird im Fall eines vorausgegangenen Autounfalls im Sinne eines Diskriminationstrainings daran gearbeitet, die vergangene Unfallsituation von der Situation im Hier und
Jetzt trennen zu lernen (König 2012). Mit den verschiedenen Techniken der kognitiven Umstrukturierung (z. B. Realitätscheck, Überprüfung der logischen Konsistenz, empirische, hedonistische und funktionale Disputation) soll an der Bearbeitung unfallbezogener Sorgen und der Entwicklung hilfreicherer Gedanken gearbeitet werden.

Steht die Facette der Angst vor der Bewertung durch andere im Vordergrund, soll der/die Patient/-in mit dieser Angst konfrontiert werden, indem durch Verhaltensexperimente Erwartungen überprüft werden und die Wirkung der nach innen gerichteten Selbstaufmerksamkeit als angstverstärkend herausgearbeitet wird (Stangier et al. 2009). Unabhängig von der Schwerpunktsetzung wird in typische Denkverzerrungen (z. B. Schwarz-Weiß-Denken, Verallgemeinerungen etc.) anhand individueller Beispiele eingeführt, was einer Umstrukturierung der autofahrbezogenen Angstkognitionen dienen soll.

Nach innen gerichtete Aufmerksamkeitsprozesse können panikbezogene Sorgen und die Sorgen vor der Bewertung durch andere (ähnlich wie überhöhtes „self-monitoring“ sozialphobischer Patienten/Patientinnen) verstärken und aufrechterhalten. Somit wird in das Konzept der Achtsamkeit eingeführt, um Aufmerksamkeitsprozesse salient zu machen und die Lenkung des Aufmerksamkeitsscheinwerfers (nach innen oder außen) zu trainieren.

\section{Motivierungsphase}

In der anschließenden Motivierungsphase wird ein Gedankenexperiment zur vertieften Auseinandersetzung mit den dysfunktionalen Erwartungen durchgeführt, in dem der/die Patient/-in dazu angeleitet wird, sich die gefürchtete $\mathrm{Au}$ tofahrsituation vorzustellen und sich so damit zu konfrontieren (in Anlehnung an Lang et al. 2018). Ziel ist, den/die Patienten/Patientin zu ermutigen, über den schlimmsten Moment hinauszudenken, eine Abnahme der Angstreduktion zu antizipieren sowie eine Überprüfung und Korrektur der Erwartungen zu erleben. Die Rationale der Konfrontation und Erwartungsüberprüfung wird hier verdeutlicht. Das Behandlungsmodul sieht an dieser Stelle die Aufklärung über mögliche Risiken bei der In-vivoKonfrontation im Straßenverkehr vor, in die die Betroffenen schriftlich einwilligen müssen. Ein Formulierungsbeispiel der Einverständniserklärung könnte wie folgt lauten und wird von Therapeut/-in und Patient/-in unterschrieben:

Der Patient/die Patientin Herr/Frau geboren am __ wohnhaft in __ wurde über die Risiken, die bei der Exposition mit Autofahrängsten im Straßenverkehr bestehen, umfänglich aufgeklärt, z. B. Einfluss von akuten Angsterscheinungen wie Herzrasen auf die Fahrtauglichkeit, und er/sie wurde auf die dadurch entstehende mögliche Eigen- und Fremdgefährdung explizit hingewiesen. Unter Berücksichtigung aller Risiken hat sich Herr/Frau mit der Expositionsbehandlung einverstanden erklärt und möchte sich dieser aussetzen.

\section{In-vivo-Exposition}

Die oben beschriebene kognitive Vorbereitung dient zur Steigerung der Bereitschaft für die In-vivo-Exposition. Die Exposition besteht, in Anlehnung an die versicherungsrechtlichen Empfehlungen, zunächst aus einer doppelstündigen Konfrontation im Fahrsimulator, dann aus einer ebenfalls doppelstündigen Exposition im Fahrschulauto mit Fahrlehrer/-in und bei gegebener Fahrtauglichkeitseinschätzung durch den/die Fahrlehrer/-in aus zwei Doppelstunden therapeutisch begleiteter Expositionen im patienteneigenen Auto. In Abhängigkeit von Fahrpraxis, Grad der bisherigen Vermeidung und der gezeigten Fahrkompetenz können mehr Fahrstunden in Begleitung des Fahrlehrers notwendig werden. Der erwartete und tatsächliche Angstverlauf wird in jeder Exposition festgehalten, mit dem/der Patienten/ Patientin reflektiert, und die Erwartungsverletzungen werden herausgearbeitet. Zwischen diesen und den folgenden Sitzungen soll der/die Patient/-in unbegleitete Expositionen durchführen. Während der Expositionsphase wird primär der Wirkmechanismus der Habituation an die erlebte Angstreaktion, sowohl innerhalb der Sitzungen und während 
der Fahrten („in-session habituation“), als auch zwischen den Sitzungen und den erfolgten Fahrten („between-session habituation"), angenommen. Sekundär ergibt sich durch die Konfrontation und die korrigierenden Erfahrungen jedoch auch die Möglichkeit zur Generierung von langfristig angstreduzierenden Erwartungsverletzungen. Bei im Vordergrund stehenden panikbezogenen Ängsten sollten möglichst die Fahrsituationen in den Expositionen aufgesucht werden, die die gefürchtete körperliche Symptomatik auslösen. Das kann z.B. das Einfädeln auf und Befahren von $\mathrm{Au}$ tobahnen sein, das Fahren mit höherer Geschwindigkeit oder das Fahren über Brücken oder durch Tunnel. Liegen hauptsächlich unfallbezogene Sorgen vor, sollten jene Strecken befahren werden, bei denen der/die Betroffene ein hohes Unfallrisiko antizipiert oder in der Vergangenheit Unfälle beobachtet und erlebt hat. Im Fall von der Angst vor der Bewertung durch andere können sich Innenstadtfahrten zur Exposition anbieten, Einparksituationen, in denen Hinterherfahrende warten müssen oder Passanten Zuschauer sind, oder das Stehen an einer roten Ampel an erster Stelle mit verzögertem Losfahren.

\section{Abschluss}

Zum Abschluss des Behandlungsmoduls findet ein Rückblick auf die hochfrequente Angstbehandlung statt; es werden Fortschritte beleuchtet und ein Umgang mit möglichen Rückschritten besprochen. Es wird ein Übungsplan erarbeitet, sodass der/die Betroffene in den kommenden 6 Wochen bis zur BoosterSitzung selbstständig üben kann. Die Erfahrungen des selbstständigen Übens werden reflektiert, Fortschritte validiert und mögliche Schwierigkeiten besprochen. Zum Abschluss nach weiteren 6 Wochen wird das Erreichte reflektiert und ein Fazit der Behandlung gezogen.

\section{Erste Erfahrungen und Ausblick}

Das vorgestellte Behandlungsvorgehen wurde durch 15 Therapeuten/ Therapeutinnen bei bereits rund 34 Patienten/Patientinnen angewendet, sodass erste Erfahrungen skizziert werden können.

Die Wichtigkeit der Beachtung der drei dargestellten Schwerpunkte im Erklärungsmodell wurde bestätigt, um auf individuelle Schwerpunkte der Autofahrangst eingehen $\mathrm{zu}$ können. So erwies sich für Personen mit panikbezogenen Sorgen beim Autofahren die Konfrontation mit Körpersymptomen im Therapieraum als zieldienlich, wodurch ein Aushalten und Beobachten der Reduktion der physiologischen Erregung beim Autofahren möglich wurde. Betroffene mit Sorgen vor der Bewertung durch andere profitierten deutlich von konkret diese Sorgen evozierenden Konfrontationsübungen, wie z. B. dem Stehen an einer roten Ampel an erster Stelle oder dem Einparken am Straßenrand bei Aufhalten der Hinterherfahrenden. Ein sehr häufige Angstsituation bei von Autofahrangst Betroffenen stellten die Angst vor dem Fahren und Überholen auf der Autobahn sowie das Auffahren auf die Autobahn dar.

Die Fahrsimulatornutzung schien für Personen mit wenig Fahrpraxis bzw. ausgeprägtem Vermeidungsverhalten sinnvoll, da so ein Kompetenz- und Fertigkeitstraining ermöglicht wurde. Das Autofahren gänzlich vermeidende Personen erprobten somit notwendige Fertigkeiten, wie die Abläufe beim Abbiegen, im sicheren Rahmen. Bei großer Skepsis vor der In-vivo-Exposition konnte mithilfe des Simulators die Bereitschaft zur In-vivo-Konfrontation gesteigert werden. Teils konnte ein gefährlicher Fahrstil (z.B. abruptes, unkontrolliertes Abfahren von der Autobahn bei aufkommender Angst) beobachtet werden, sodass im Simulator ein geeigneter, sicherer Übungsrahmen entstand. Autofahrängstliche „Vielfahrer", die lediglich spezifische Einzelsituationen (z.B. das Fahren bei Nacht oder bei Glätte) fürchteten, profitierten weniger von der Simulatornutzung, da sich diese als nicht spezifisch genug herausstellte. Einige Patienten/Patientinnen litten während der Fahrt im Simulator unter „motion sickness“, was z.T. auf die Unerfahrenheit mit dem Bewegen in virtuellen Realitäten zurückzuführen war. Anstelle eines Fahrsimulators, der häufig nicht vorhanden ist, kann das Betrachten einzelner Videosequenzen von Fahrsituationen aus der Perspektive des Fahrers denkbar sein.

Der Einbezug der Fahrschule scheint unumgänglich, da somit die versicherungsrechtlichen Aspekte eingehalten werden und die durch Expositionen im Straßenverkehr entstehenden Gefahren für Patient/-in und Therapeut/-in deutlich minimiert werden können. Die Fahrkompetenz wird durch den Einbezug der Fahrschule nicht nur überprüft, sondern in vielen Fällen sogar gesteigert, da Rückmeldungen seitens des Fahrlehrers auf typische Fahrfehler hinweisen (z.B. zu langsames und zögerliches Auffahren auf Autobahnen), das Üben und die Konfrontationen präzisieren sowie die autofahrbezogene Selbstwirksamkeit langfristig stärken können.

Das beschriebene graduierte Vorgehen mit Einbezug eines Simulators, des/der Fahrlehrers/-in und schließlich des/der Therapeuten/-in als Beifahrer/-in kann hinsichtlich seiner möglichen angstaufrechterhaltenden Wirkung im Sinne von Sicherheitsverhaltensweisen diskutiert werden. Wenngleich im lerntheoretischen Sinne eine Beeinträchtigung des Lerneffekts bei Hinzunahme dieser Sicherheitssignale befürchtet werden kann, ergibt sich aufgrund des geschilderten Sicherheits- und Versicherungsaspekts sowie aus Gründen der neben der Angst potenziell bestehenden Kompetenzdefizite die Notwendigkeit zum graduierten Vorgehen und ggf. dem zeitweisen Einsatz von Angstbewältigungsstrategien. Aufgrund des Ausschleichens dieser sicherheitgebenden Faktoren, sowohl innerhalb des graduierten Ablaufs als auch am Ende der Behandlung in der Phase des eigenständigen Übens, halten die Autoren das Vorgehen lerntheoretisch und verhaltenstherapeutisch nicht prinzipiell für den Therapieeffekt minimierend (Telch und Lancaster 2012).

Insgesamt zeigt der aktuelle Stand der Wissenschaft noch viel Forschungsbedarf im Bereich der Ätiologie, Diagnostik und Therapie von Autofahrangst. Eine Veränderung der technischen Möglichkeiten, wie die Entwicklung des autonomen Fahrens, könnte in Zukunft zu einer 
veränderten Form der Angst hinter dem Steuer führen und ihrerseits neue Forschungsfragen aufwerfen.

\section{Fazit für die Praxis}

\section{- Diagnosestellung und Therapiepla- nung werden durch die Herausar- beitung der individuell spezifischen Ätiologiefaktoren der Autofahrangst verbessert. \\ - Die Aufklärung über Risiken, die Einwilligung des/der Patienten/ Patientin und ihre Dokumentation müssen vor der In- vivo-Konfrontati- on im Straßenverkehr erfolgen. \\ - Eine Exposition sollte graduiert er- folgen, z. B. unter Hinzunahme einer Fahrschule. Dazu bedarf es Koope- rationen mit über die Rationale der Exposition informierten Fahrleh- rern/Fahrlehrerinnen, damit es nicht zu therapeutisch gegenläufigen Instruktionen kommt (z. B. Beruhi- gung durch den/die Fahrlehrer/-in). Durch ihn/sie wird eine Fahrtauglich- keitseinschätzung vorgenommen. \\ - Für eine anschließende Exposition im Straßenverkehr im patienteneigenen Auto ist eine Berufshaftpflichtver- sicherung, die Schäden durch den Betrieb von Kraftfahrzeugen ab- deckt, notwendige Voraussetzung.}

\section{Korrespondenzadresse}

\section{Carolin Fischer, M.Sc.}

Arbeitseinheit für Klinische Psychologie und Psychotherapie des Erwachsenenalters, Universität Koblenz-Landau Ostbahnstr. 10, 76829 Landau, Deutschland fisc8458@uni-landau.de

Funding. Open Access funding enabled and organized by Projekt DEAL.

\section{Einhaltung ethischer Richtlinien}

Interessenkonflikt. C. Fischer, A. Schröder und J. Heider geben an, dass kein Interessenkonflikt besteht.

Alle beschriebenen Untersuchungen am Menschen wurden mit Zustimmung der zuständigen Ethik-Kommission, im Einklang mit nationalem Recht sowie gemäß der Deklaration von Helsinki von 1975 (in der aktuellen, überarbeiteten Fassung) durchgeführt. Von allen beteiligten Patienten liegt eine Einverständniserklärung vor.
Open Access. Dieser Artikel wird unter der Creative Commons Namensnennung 4.0 International Lizenz veröffentlicht, welche die Nutzung, Vervielfältigung, Bearbeitung, Verbreitung und Wiedergabe in jeglichem Medium und Format erlaubt, sofern Sie den/die ursprünglichen Autor(en) und die Quelle ordnungsgemäß nennen, einen Link zur Creative Commons Lizenz beifügen und angeben, ob Änderungen vorgenommen wurden.

Die in diesem Artikel enthaltenen Bilder und sonstiges Drittmaterial unterliegen ebenfalls der genannten Creative Commons Lizenz, sofern sich aus der Abbildungslegende nichts anderes ergibt. Sofern das betreffende Material nicht unter der genannten Creative Commons Lizenz steht und die betreffende Handlung nicht nach gesetzlichen Vorschriften erlaubt ist, ist für die oben aufgeführten Weiterverwendungen des Materials die Einwilligung des jeweiligen Rechteinhabers einzuholen.

Weitere Details zur Lizenz entnehmen Sie bitte der Lizenzinformation auf http://creativecommons.org/ licenses/by/4.0/deed.de.

\section{Literatur}

Becker ES, Rinck M, Türke V, Kause P, Goodwin R, Neumer S, Margraf J (2007) Epidemiology of specific phobia subtypes: findings from the Dresden Mental Health Study. Eur psychiatr 22:69-74

da Costa R, de Carvalho M, Ribeiro P, Nardi A (2018) Virtual reality exposure therapy for fear of driving. Rev Bras Psiquiatr 40(2):192-199

Deutsche Psychotherapeuten Vereinigung (2016) Expositionsbehandlung - BehandlungsfehlerHaftung

Ehlers A, Hofmann SG, Herda CA, Roth WT (1994) Clinical characteristics of driving phobia. JAnxiety Disord 8(4):323-339

Ehring T, Ehlers A, Glucksman E (2006) Contribution of cognitive factors to the prediction of posttraumatic stress disorder, phobia and depression after motor vehicle accidents. Behav Res Ther 44(12):1699-1716

Emmelkamp P, Bruynzeel M, Drost L, van der Mast C (2001) Virtual reality treatment in acrophobia: a comparison with exposure in vivo. Cyber Psychol Behav 4(3):335-339

Fischer C, Heider J, Schröder A, Taylor JE (2020) “Help! I'm afraid of driving!" review of driving fear and its treatment. Cogn Ther Res 44(2):420-444

Fischer C, Schröder A, Heider J Cognitive behavior therapy for driving fear: a randomized controlled pilot study. (in Vorbereitung)

Fischer C, Schröder A, Taylor JE, Heider J Measuring driving fear: development and validation of the instrument for fear of driving (IFD). (under review)

Halbe B (2003) Haftungsgrundsätze für Psychotherapeuten bei der Expositionsbehandlung, insbesondere bei Patienten mit Angststörungen im Straßenverkehr. Forum Psychother Prax 3(4):173-179

Heider J, Fischer C, Schröder A (2018) Die deutsche Version des "Driving Cognitions Questionnaire (DCQ).Z Klin Psychol Psychother 47(1):36-47

Horn S, Heider J, Seibert-Schleich A, Schröder A (2015) Highway to hell - Expositionstherapie bei Autofahrphobie: Ein Fallbericht. Verhaltensther Verhaltensmed 36(1):50-60
KaussnerY, Kuraszkiewicz AM, SchochS, MarkelP, Hoffmann S, Baur-Streubel R, Kenntner-Mabiala R, Pauli P (2020) Treating patients with driving phobia by virtual reality exposure therapy - a pilot study. PLoSONE 15(1):1-14

König J (2012) Posttraumatische Belastungsstörung: Ein Manual zur Cognitive Processing Therapy. Hogrefe, Göttingen

Kuch K, Swinson R, Kirby M (1985) Post-traumatic stress disorder after car accidents. Can J Psychiatry 30(6):426-427

Lang T, Helbig-Lang S, Westphal D, Gloster A, Wittchen $\mathrm{H}-\mathrm{U}$ (2018) Expositionsbasierte Therapie der Panikstörung mit Agoraphobie. Hogrefe, Göttingen

Munjack DJ (1984) The onset of driving phobias. JBehav Ther Exp Psychiatry 15(4):305-308

Pittig A, Hoyer J (2017) Exposition aus Sicht niedergelassener Verhaltenstherapeutinnen und Verhaltenstherapeuten: Anwendung und systemische Barrieren. Z Klin Psychol Psychother 46(4):223-235

Ruhmland M, Margraf J (2001) Effektivität psychologischer Therapien von spezifischer Phobie und Zwangsstörung: Meta-Analysen auf Störungsebene. Verhaltenstherapie 11(1):14-26

Stangier U, Heidenreich T, Peitz M (2009) Soziale Phobien: Ein kognitiv-verhaltenstherapeutisches Behandlungsmanual. Beltz, Weinheim

Taylor J, Deane F (1999) Acquisition and severity of driving-related fears. Behav Res Ther 37(5):435-449

Taylor J, Deane F, Podd J (1999) Stability of driving fear acquisition pathways over one year. Behav Res Ther 37(10):927-939

Taylor J, Deane F, Podd J (2000) Determining the focus of driving fears. J Anxiety Disord 14(5):453-470

Taylor J, Deane F, Podd J (2002) Driving-related fear: a review. Clin Psychol Rev 22:631-645

Telch M, Lancaster C (2012) Is there room for safety behaviors in exposure therapy for anxiety disorders? In: Exposure therapy. Springer, New York, S313-334

Tintner H (2002) Das Haftungsrecht des niedergelassenen Psychotherapeuten. Psychother Prax 2(1):39-44

Townend M, Grant A (2006) Integrating science, practice and reflexivity-cognitive therapy with driving phobia. J Psychiatr Ment Health Nurs 13(5):554-561 